\title{
HAAR MEASURE AND THE SEMIGROUP OF MEASURES ON A COMPACT GROUP
}

\author{
J. G. WENDEL
}

1. Introduction. Crucial results in the theory of a compact topological semigroup $S$ state that $S$ must possess idempotents, and that if $S$ contains an identity and is not a group then at least one additional idempotent occurs $[6 ; 7 ; 8]$. These facts were pointed out to the author by R. J. Koch, whom it is here a pleasure to thank for many stimulating and instructive conversations.

An interesting example is furnished by the set $S$ of (normalized nonnegative regular) measures on a compact group $G$; $S$ becomes a compact Hausdorff semigroup with identity under natural definitions of multiplication and topology, and $S$ is not a group if $G$ has more than one element. Then $S$ has additional idempotents; what are they? If the existence of Haar measures is assumed it is easy to show that the Haar measure associated with any compact subgroup of $G$ determines an idempotent. The converse is more interesting, and is our main concern here: the existence of idempotents in $S$ implies the existence of Haar measure on $G$. (At this writing we are unable to apply the method to general locally compact groups.) As a secondary result we show that $G$ is determined by $S$.

2. Definitions and preliminary results. Let $G$ be a compact group and let $S$ be the set of countably additive nonnegative regular set functions $\mu$ on the Borel sets of $G$ with $\mu(G)=1$. Let $C(G)$ be the Banach space of real continuous functions on $G$, and recall the 1-1 correspondence between measures $\mu \in S$ and continuous linear functionals $\phi$ on $C(G)$, with the properties $f(x) \geqq 0$ implies $\phi(f) \geqq 0$ and $\phi(1)=1$, that is given by the equation $\phi(f)=\int f(x) d \mu(x)$. For later reference we note the formula (cf. [3, Theorem 56. E])

$$
\mu(U)=\sup \left\{\int f(x) d \mu(x) \mid f \in C(G)\right.
$$

$$
0 \leqq f(x) \leqq 1, f(x)=0 \text { for } x \notin U\}
$$

valid for all open sets $U$ in $G$.

We give $S$ the weak* topology for functionals, so that $\mu_{\alpha} \rightarrow \mu$ means

Presented to the Society, February 27, 1954; received by the editors January 11, 1954 and, in revised form, March 15, 1954. 
$\int f(x) d \mu_{\alpha}(x) \rightarrow \int f(x) d \mu(x), f \in C(G) . S$ is compact in this topology, by virtue of the weak* compactness of the unit sphere in the conjugate space of $C(G)$ and the fact that the restrictions on $\phi$ 's imposed above determine a closed subset of the unit sphere.

As in [2] we define multiplication of $\mu, \nu \in S$ by

$$
\int f(x) d(\mu \nu)(x)=\iint f(y z) d \mu(y) d \nu(z), \quad f \in C(G) ;
$$

it is easy to see that the multiplication is associative and continuous on $S$. Thus $S$ is a compact topological semigroup.

Now for any element $x \in G$, we define the element $x^{\prime} \in S$ as the point mass at $x$, i.e. $x^{\prime}(E)=1$ if $x \in E, 0$ if $x \in E$. The corresponding functional sends the function $f$ into the number $f(x)$, and the group element $x y$ goes over into the measure $(x y)^{\prime}=x^{\prime} y^{\prime}$. Therefore the mapping $x \rightarrow x^{\prime}$ of $G$ into $S$ is a homeomorphic isomorphism, so that henceforth we may regard $G$ as embedded in $S$ and omit primes. Clearly the identity $e$ of $G$ is also the identity for $S$; in fact, making use of (2) it is easy to see that for $x \in G$

$$
(\mu x)(E)=\mu\left(E x^{-1}\right), \quad(x \mu)(E)=\mu\left(x^{-1} E\right),
$$

all $E$.

The semigroup $S$ has two special structural features not shared by all compact semigroups with identity, which are: the possibility of forming convex linear combinations of elements of $S$, and the presence of a natural antiautomorphic involution in $S$; it is doubtful whether we have made maximal use of these properties, but at any rate they turn out to be very useful.

More explicitly, for $\mu, \nu \in S$ and $t$ real, $0 \leqq t \leqq 1, t \mu+(1-t) \nu$ is again in $S$. This remark enables us to exhibit elements of $S$ which do not possess inverses if $G$ has more than one element, so that $S$ is not $a$ group. In fact, for $x, y \in G$ the measure $(1 / 2) x+(1 / 2) y$ has no inverse; this is a consequence of the following lemma whose proof we defer to the next section.

Lemma 1. A necessary and sufficient condition that $\mu \in S$ have an inverse is that $\mu$ is a point mass, $\mu \in G$.

From this it follows easily that $S$ determines $G$, for in order to reconstruct $G$ from $S$ we have only to pick out the elements with inverses.

The involution $\mu \rightarrow \mu^{*}$ is defined by $\mu^{*}(E)=\mu\left(E^{-1}\right)$; the assertion $\mu^{* *}=\mu$ is trivial, and the fact that $(\mu \nu)^{*}=\nu^{*} \mu^{*}$ follows easily from $\int f(x) d \mu^{*}(x)=\int f\left(x^{-1}\right) d \mu(x)$ and equation (2). A deeper fact about the involution is contained in 
LEmma 2. If $\mu$ is idempotent, then $\mu^{*}=\mu$.

Again the proof is postponed to the next section.

3. The idempotents of $S$. Let $H$ be a compact subgroup of $G$ and suppose that $H$ has Haar measure $\mu$. We extend the definition of $\mu$ to all the Borel sets of $G$ by putting $\mu(E)=\mu(E \cap H)$, and show that $\mu$ is idempotent. This follows from the more general statement that if $\nu \in S$ is carried on $H$ (this means that $\nu(H)=1$ ), then $\mu$ annihilates $\nu, \mu \nu=\nu \mu=\mu$. In fact, to show that $\mu \nu=\mu$ we have $\int f(x) d(\mu \nu)(x)$ $=\iint f(y z) d \mu(y) d \nu(z)=\int_{H} \int_{H} f(y z) d \mu(y) d \nu(z)=\int_{H} \int_{H} f(y) d \mu(y) d \nu(z)$ $=\left\{\int_{H} f(y) d \mu(y)\right\} \nu(H)=\int f(x) d \mu(x), f \in C(G)$, and similarly $\nu \mu=\mu$.

We remark two important special cases: if $H=\{e\}$ then $\mu=e$, and if $H=G$ then $\mu=$ Haar measure of $G=$ annihilator of $S$. Of course here we are assuming that $G$ has Haar measure.

Conversely, without assuming the existence of Haar measure, we are going to prove

THEOREM 1. Let $\mu$ be an idempotent in $S$. There exists a unique compact subgroup $H$ in $G$ such that $\mu$ is the Haar measure of $H$, extended as above to all of $G$.

Before proving the theorem we need some lemmas, as follows.

Lемма 3. For any $\mu \in S$ there is a unique closed set $A$, the carrier of $\mu$, such that $\mu(A)=1$ and $\mu(U)>0$ for any nonempty relatively open subset $U$ of $A$.

Proof. The uniqueness of $A$ is easy, for if $A^{\prime}$ were a second closed set with the specified properties we could choose the notation so that $U=A^{\prime}-A$ would be nonempty and write

$$
1=\mu(G) \geqq \mu(A)+\mu\left(A^{\prime}-A\right)=1+\mu\left(A^{\prime}-A\right)>1,
$$

a contradiction.

For the proof of existence let $\mathcal{F}$ be the family of closed sets $F$ with $\mu(F)=1 . G \in \mathcal{F}$; hence $\mathcal{F}$ is not empty. For $F_{1}, F_{2}, \cdots, F_{n} \in \mathcal{F}$ we find that $\bigcap_{i} F_{i}$ again belongs to $\mathcal{F}$, since the complements of the $F_{i}$ are $\mu$-nullsets. Thus $\mathcal{Z}$ has the finite intersection property; let $A$ be the closed nonempty set of points common to all the $F^{\prime}$ s. Let $U$ be an open set containing $A$. Then $U$ contains some finite intersection of $F$ 's. Therefore $\mu(U)=1$. Then the regularity of $\mu$ shows that $\mu(A)=1$. No closed proper subset of $A$ belongs to $\mathcal{F}$, i.e. has $\mu$-measure equal to 1 , and this observation completes the proof of the lemma.

Lemma 4. Let $A$ and $B$ be the carriers of $\mu$ and $\nu$. Then $A B$ $=\{x y \mid x \in A, y \in B\}$ is the carrier of $\mu \nu$. 
We shall first show that the desired relation follows from

$$
(\mu \nu)(V W) \geqq \mu(V) \nu(W), \quad V \text { and } W \text { open in } G .
$$

Let $U$ be an open set containing $A B$; by the continuity of multiplication we may find open $V$ and $W$ containing $A$ and $B$ such that $V W$ is contained in $U$. Then

$$
(\mu \nu)(U) \geqq(\mu \nu)(V W) \geqq \mu(V) \nu(W) \geqq \mu(A) \nu(B)=1 ;
$$

hence by the regularity of $\mu \nu,(\mu \nu)(A B)=1$. Similarly, if $U$ is open in $A B$ it follows easily that $(\mu \nu)(U)>0$. Therefore $A B$, which is closed by a known theorem, has the required properties.

It remains to establish (3), which we shall do by invoking (1). Let $g$ and $h$ be continuous maps of $G$ to $[0,1]$ vanishing outside of $V$ and $W$ respectively. Define a real function $f$ on $G$ by $f(z)=\max _{x} g(x) h\left(x^{-1} z\right)$. Clearly $f$ is continuous, $f$ sends $G$ to $[0,1]$, and $f$ vanishes outside of $V W$. Moreover, $f(x y) \geqq g(x) h(y)$. Thus $\int f(z) d(\mu \nu)(z)$ $=\iint f(x y) d \mu(x) d \nu(y) \geqq \int g(x) d \mu(x) \int h(y) d \nu(y)$; taking suprema over $g$ and $h$ and applying (1) then yields (3).

Proof of Lemma 1. If $\mu \nu=e$ and $A, B$ are the carriers of $\mu, \nu$, then $A B=\{e\}$. Hence $A$ and $B$ are one-point subsets of $G$, as had to be shown.

Lemma 5. If $\mu$ is idempotent and $H$ its carrier, then $H$ is a compact subgroup of $G$.

Proof. By Lemma $4, H^{2}=H$. Therefore by a known theorem [ 1 ; 6] $H$ is a compact subgroup.

Proof of Theorem 1. Let $H$ be the carrier of $\mu$. By Lemma 3 every open set of $H$ has positive $\mu$-measure and, by Lemma $5, H$ is a compact subgroup of $G$. To show that $\mu$ is translation-invariant on $H$ we must show that for any $f \in C(H)$ the expression

$$
g(y)=\int f(x y) d \mu(x)
$$

is constant for $y \in H$. The function $g$ is continuous and attains its maximum at a point which, after replacing $f$ by a suitable translate, we may take to be $y=e$. Then from (2) we have

$$
\begin{aligned}
g(e)=\int f(x) d \mu(x) & =\iint f(y z) d \mu(y) d \mu(z)=\int g(z) d \mu(z) \\
& \leqq \int g(e) d \mu(z)=g(e)
\end{aligned}
$$


Therefore $g$ is identically $g(e)$, and since the uniqueness of $H$ is trivial Theorem 1 is proved.

Proof of Lemma 2. This is an easy consequence of Theorem 1 and the known fact applied to $H$ that on a compact group Haar measure is invariant under inverse.

4. The existence of Haar measure. Now we want to apply the foregoing considerations to give a new proof of the existence of Haar measure for compact groups. Let $G \neq\{e\}$; then, as we have seen, $S$ is a compact semigroup with identity, which is not a group. There is then another idempotent in $S$, and by Theorem 1 some nontrivial subgroup $H$ of $G$ has Haar measure. The idea of the proof is to show first that there is a largest such subgroup $H$, which is necessarily normal in $G$; then if $H$ is not already $G$ we shall show that we have a contradiction.

We begin by partially ordering the compact subgroups $H$ of $G$ which have Haar measure under inclusion. The results of the previous section show that this is equivalent to partially-ordering the idempotents of $S$ by annihilation: $\mu \leqq \nu$ means $\mu \nu=\nu \mu=\nu$.

LemMa 6. The set of idempotents is a directed set in the above ordering.

Proof. Let $\mu, \nu$ be distinct idempotents of $S$. Form $\sigma=\mu \nu$ and let $T$ be the closure in $S$ of the set of powers of $\sigma$. It is clear that $T$ is a compact subsemigroup of $S$. Since $\mu \sigma^{n}=\sigma^{n} \nu=\sigma^{n}$ we have $\mu \tau=\tau \nu=\tau$ for $\tau \in T$. (If $G$ (and therefore $S$ ) is abelian, then trivially $T=\{\sigma\}$ $=\{\mu \nu\}$.) By the fundamental theorem there is an idempotent $\tau$ in $T$. Applying the involution and Lemma 2 we find that $\tau$ annihilates both $\mu$ and $\nu$. That is, $\tau \geqq \mu, \tau \geqq \nu$. (Recent results of Koch [4] show that $\tau$ is unique; from this it follows easily that the carrier of $\tau$ is the closed subgroup generated by the carriers of $\mu$ and $\nu$.)

Lemma 7. There is a greatest idempotent in $S$.

PROOF. The set of idempotents is defined by the relation $\mu^{2}=\mu$ and therefore is compact. For each $\mu$ let $Q(\mu)=\{\nu \mid \nu \geqq \mu\}$. These sets are closed, since they are defined by relations $\nu^{2}=\nu=\nu \mu=\mu \nu$. By Lemma 6 the family of $Q(\mu)$ 's has the finite intersection property, and hence there is a point common to all of them. Another application of Lemma 6 shows that there is precisely one such point, and this is the desired greatest idempotent.

Leмma 8. Let $H$ be the carrier of the greatest idempotent $\mu$. Then $H$ is a nontrivial compact normal subgroup of $G$.

Proof. $H$ is a compact subgroup of Theorem 1: $H \neq\{e\}$ since $e$ is 
certainly not the greatest idempotent. Finally we show that $H$ is normal. Let $x \in G$. Then the measure $x^{-1} \mu x$ is idempotent, and its carrier is $x^{-1} H x$, by Lemma 4 . We must have $x^{-1} \mu x=\mu$ with the consequent $x^{-1} H x=H$, since otherwise $\mu>x^{-1} \mu x$, from which it would easily follow that $x \mu x^{-1}>\mu$, a contradiction.

\section{TheOREM 2. G has Haar measure.}

Proof. If $H$ of Lemma 8 is $G$ we are finished. But if $H \neq G$ we can construct an idempotent greater than $\mu$ in the following way. As shown in the proof of Lemma $8, \mu$ commutes with each element of $G$. Then also $\mu$ commutes with each $\nu \in S$, for $\iint f(x y) d \mu(x) d \nu(y)$ $=\iint f\left(y x y^{-1} y\right) d \mu\left(y x y^{-1}\right) d \nu(y)=\iint f(y x) d \mu(x) d \nu(y)=\iint f(x y) d \nu(x) d \mu(y)$. Let $S^{\prime}=S \mu$, a compact semigroup with identity $\mu$. $S^{\prime}$ is not a group, for if $x \notin H$ the element $(1 / 2) x \mu+(1 / 2) \mu$ can have no inverse in $S^{\prime}=S \mu$, by an argument with carriers similar to that used in proving Lemma 1. Then $S^{\prime}$ contains an idempotent $\nu=\nu \mu \neq \mu$, and $\mu \nu \mu=\nu \mu \mu$ $=\nu \mu>\mu$. This contradiction completes the proof.

It may be worthwhile to sketch the mechanism underlying this proof. One might attempt to prove Theorem 2 as follows. If $H \neq G$, form the compact group $G^{\prime}=G / H$, and find therein a nontrivial subgroup $K^{\prime}$ having Haar measure; by a modification of a device used in $[3 ; \mathbf{5}]$ one can then construct Haar measure on $K$, the antecedent of $K^{\prime}$ in the natural map of $G$ upon $G^{\prime} ; K$ properly contains $H$, a contradiction. The precise connection between these remarks and the given proof is found in

Theorem 3. $S^{\prime}=S \mu$ may be identified with the semigroup $S\left(G^{\prime}\right)$ of measures on $G^{\prime}=G / H$. (This does not depend on maximality of $H$, but only on its normality in $G$.)

Proof. Let $\nu^{\prime} \in S\left(G^{\prime}\right)$ and $f \in C(G)$. Form $F(x)=\int f(x y) d \mu(y)$ $=\iint f(x y z) d \mu(y) d \mu(z) . F$ is continuous and is constant on cosets $x^{\prime}$ of $H$ and therefore defines an element of $C\left(G^{\prime}\right)$. Form $\int F(x) d \nu^{\prime}\left(x^{\prime}\right)$, where $x$ is any element of $x^{\prime}$. This defines a functional of the apappropriate kind on $C(G)$, and so may be written in the form $\int f(x) d \nu(x)=\iint f(x z) d \nu(x) d \mu(z)$ with $\nu \in S$. Hence $\nu=\nu \mu \in S \mu=S^{\prime}$. It is routine to verify that the mapping $\nu^{\prime} \rightarrow \nu \mu$ is a continuous (and therefore homeomorphic) isomorphism of $S\left(G^{\prime}\right)$ onto $S^{\prime}$; we suppress further details.

\section{BiBLIOGRAPHY}

1. B. Gelbaum, G. K. Kalisch, and J. M. H. Olmsted, On the embedding of topological semigroups and integral domains, Proc. Amer. Math. Soc. vol. 2 (1951) pp. 807 821. 
2. R. Godement, Les fonctions de type positif et la theorie des groupes, Trans. Amer. Math. Soc. vol. 63 (1948) pp. 1-84.

3. P. R. Halmos, Measure theory, New York, 1950.

4. R. J. Koch, Tulane University Dissertation, 1953.

5. L. H. Loomis, An introduction to abstract harmonic analysis, New York, 1953.

6. K. Numakura, On bicompact semigroups, Mathematical Journal of Okayama University vol. 1 (1952) pp. 98-100.

7. J. E. L. Peck, Yale University Dissertation, 1950.

8. A. D. Wallace, $A$ note on mobs, Anais da Academia Brasileira de Ciencias vol. 24 (1952) pp. 329-334.

Louisiana State University

\section{ON THE SPECTRA OF COMMUTATORS ${ }^{1}$}

\section{R. PUTNAM}

1. The following theorems will be proved:

$\left({ }^{*}\right)$ Let $A$ and $B$ denote bounded operators in a Hilbert space and suppose that the commutator

$$
C=A B-B A
$$

satisfies the commutation relation

$$
A C=C A \text {. }
$$

Then there exists a sequence of bounded operators $C_{1}, C_{2}, \cdots$ such that

$$
\left\|C-C_{n}\right\| \rightarrow 0 \text { and } \operatorname{sp}\left(C_{n}\right) \rightarrow 0 \text { as } n \rightarrow \infty .
$$

$\left({ }^{* *}\right)$ If, in addition to the assumption (2) of $(*)$, the relation

$$
B C=C B
$$

also holds, then the assertion (3) can be improved to

$$
\mathrm{sp}(C) \text { consists of } 0 \text { alone. }
$$

For the terminology see, e.g., [3, pp. 9-10]. It is understood that sp $(D)$ denotes the set of points in the spectrum of an operator $D$. Thus, the relation sp $\left(C_{n}\right) \rightarrow 0$ means that for any $\epsilon>0$ there is a number $N_{\epsilon}$ such that the spectrum of $C_{n}$ is contained in the disk $|\lambda|<\epsilon$ whenever $n>N_{\text {e. }}$

As a consequence of $\left({ }^{* *}\right)$ one obtains the

Corollary. If $A$ is a bounded operator in a Hilbert space which com-

Received by the editors March 23, 1954.

1 This work was supported in part by the National Science Foundation research grant NSF-G481. 